\title{
Cochlear Implantation and Single-sided Deafness: A Systematic Review of the Literature
}

\author{
Francisco Cabral Junior ${ }^{1}$ Mariana Hausen Pinna ${ }^{1}$ Ricardo Dourado Alves ${ }^{1}$ \\ Andrea Felice dos Santos Malerbi ${ }^{1}$ Ricardo Ferreira Bento ${ }^{1}$ \\ ${ }^{1}$ Department of Otolaryngology, Universidade de São Paulo, \\ São Paulo, SP, Brazil \\ Int Arch Otorhinolaryngol 2016;20:69-75. \\ Address for correspondence Francisco Cabral Junior, MD, Department \\ of Otolaryngology, Universidade de São Paulo, Av. Dr. Enéas de \\ Carvalho Aguiar, 255 - $6^{\circ}$ andar - sala 6167, São Paulo, Brazil 05403-000 \\ (e-mail: fcabral@yahoo.com.br).
}

\begin{abstract}
Keywords

- unilateral hearing loss

- cochlear implantation

- single-sided deafness

- speech discrimination

- sound localization

- tinnitus

Introduction Current data show that binaural hearing is superior to unilateral hearing, specifically in the understanding of speech in noisy environments. Furthermore, unilateral hearing reduce onés ability to localize sound.

Objectives This study provides a systematic review of recent studies to evaluate the outcomes of cochlear implantation in patients with single-sided deafness (SSD) with regards to speech discrimination, sound localization and tinnitus suppression.

Data Synthesis We performed a search in the PubMed, Cochrane Library and Lilacs databases to assess studies related to cochlear implantation in patients with unilateral deafness. After critical appraisal, eleven studies were selected for data extraction and analysis of demographic, study design and outcome data.

Conclusion Although some studies have shown encouraging results on cochlear implantation and SSD, all fail to provide a high level of evidence. Larger studies are necessary to define the tangible benefits of cochlear implantation in patients with SSD.
\end{abstract}

\section{Introduction}

Single-sided deafness (SSD) may cause many problems involving communication between people. Permanent acquired unilateral several-profound hearing loss has been estimated to affect between 12-27 persons in every 100,000 among the general population, with the majority of losses being sudden or idiopathic. ${ }^{1}$ The most common impairment is difficulty in hearing sounds in the affected side due to the head shadow effect, which attenuates the highfrequency components of sounds at the ear contra-lateral to their source. ${ }^{2}$ Other problems involved are: prejudice in word discrimination, difficulty in understanding speech particularly in noisy environments; constantly adjusting head to try and compensate for the handicap; restriction of onés ability to localize sounds; and, in some cases, leading to social isolation. $^{3}$

The cochlear implant $(\mathrm{CI})$ is one of the more recent treatment options for such cases. However, there is a concern about the ability of the brain distinguish acoustic and electric stimuli and concern that the hearing from the cochlear implant would interfere with acoustic signal processing from the good ear. Contralateral routing of sound (CROS) and osseointegrated implants are also devices used as rehabilitative options for SSD, although they are not able to provide binaural hearing ${ }^{4}$ or improve sound localization. $^{5}$

People with binaural hearing enjoy certain advantages. The first advantage is better speech-to-noise ratio (SNR), which improves speech understanding in noisy environments. A second advantage results from the processing of the input sound signal by the brain from both ears. The brain is able to separate noise and speech from different locations using distinct interaural timing, spectral cues and level, thus, refining intelligibility. A third possible advantage is related to the summation effect, responsible for improved speech perception through the identification of identical signals arriving in both ears. ${ }^{6}$ received

March 11, 2015

accepted

June 2, 2015

published online

July 29, 2015
DOI http://dx.doi.org/

10.1055/s-0035-1559586. ISSN $1809-9777$.
Copyright $\odot 2016$ by Thieme Publicações License terms Ltda, Rio de Janeiro, Brazil 
Based on these facts, the use of rehabilitation methods that can restore bilateral auditory input could lead to an improvement in spatial hearing and speech perception in patients with SSD.

\section{Review of Literature}

\section{Methods}

In this article, we present some studies and reviews up to February, 2015, that analyze the influence of cochlear implantation in patients with SSD with regards to (a) sound localization, (b) speech perception, (c) tinnitus, and, (d) quality of life.

\section{Search Strategy}

A systematic search was performed in the PubMed, Embase Cochrane Library, and Lilacs databases leading up to February, 2015, using the following terms: SSD (or synonyms see - Table 1) and cochlear implantation.

\section{Study Selection}

While screening titles and abstracts, the authors excluded any duplicates, review articles, animal studies, case reports and articles written in languages other than English or Spanish. Studies published only in abstract were also excluded.

The inclusion criteria consisted of studies that analyzed patients with unilateral deafness that had undergone ipsilateral cochlear implantation, in the presence of normal or functional hearing in the contralateral ear. Implantations due to unilateral tinnitus were also included. Asymmetric hearing loss was not used as a keyword, as there is no international consensus on the definition.

\section{Results}

The search in PubMed, Cochrane Library, and Lylacs retrieved a total of 228 articles, but only 17 met the inclusion criteria and were included in the study. Next, the respective studies were appraised, according to evidence-based guidelines of categorization of medical studies (- Table $\mathbf{2}$ ), and systemati- cally analyzed. None of the studies were conducted as a randomized controlled trial and only one evaluated a control group. ${ }^{7}$ Furthermore, blinding was not observed in any study selected. Only prospective comparative studies and case series were to be analyzed in this review.

The operated patients' demographics and audiometric data were carefully examined to avoid double counting of cases. Three studies presented data which were also showed in more recent articles, including this review ${ }^{8-10}$; thus, they were discarded. Two studies scored low in patient population and did not provide suitable follow-up (patients had missed follow-up) ${ }^{11,12}$. Some studies presented incomplete data ${ }^{13}$ and were excluded for further analysis.

Therefore, after quality assessment and ruling out those failing to meet inclusion criteria, only 11 studies remained for data extraction and analysis ( - Table 3 ). All studies accepted evaluated the effect of cochlear implantation on at least one outcome of interest.

\section{Data Extraction}

A total of 137 patients with single sided-deafness have been submitted to a cochlear implant. Pooling of data was not possible due to clinical heterogeneity among the studies. Furthermore, distinct parameters were used regarding duration of deafness, indication of cochlear implant, outcome measures, and follow-up time. As described above, data are summarized in - Table $\mathbf{3}$. Several $p$ values are missing because they were not reported.

\section{Sound Localization}

In individuals with two functioning cochleae, the auditory pathway uses interaural timing and intensity variation to calculate the coordinates and localize sound correctly. For frequencies below $800 \mathrm{~Hz}$, the auditory system relies mainly on phase delays caused by interaural time differences ${ }^{14}$; whereas for frequencies greater than $1600 \mathrm{~Hz}$, it primarily relies on interaural level differences. Both phenomena are used in the transition zone from $800 \mathrm{~Hz}$ to $1600 \mathrm{~Hz}{ }^{15}$

Table 1 Search databases and filters

\begin{tabular}{|l|l|l|l|}
\hline Database & Search & Terms & Results \\
\hline PubMed & $\# 1$ & Single-sided OR one-sided OR unilateral OR monoaural & 162 \\
\hline & $\# 2$ & Deafness OR hearing loss OR loss of hearing & - \\
\hline & $\# 3$ & Cochlear implant & - \\
\hline Cochrane & $\# 1$ & S1 AND \#2 AND \#3 & - \\
\hline & $\# 2$ & Unilateral hearing loss & 8 \\
\hline & $\# 3$ & Cochlear implant & - \\
\hline & $\# 4$ & \#1 AND \#3 OR \#2 AND \#3 & - \\
\hline Scielo & $\# 1$ & $\begin{array}{l}\text { Search strategy designed for Scielo in Title/abstract fields - “unilateral hearing loss," } \\
\text { “single-sided deafness," cochlear implant }\end{array}$ & - \\
\hline
\end{tabular}


Table 2 Levels of evidence in medical research in studies that investigate therapy

\begin{tabular}{|l|l|}
\hline Level & Study design \\
\hline Level I & - Systematic review (with homogeneity) of randomized control trials (RCT) \\
& - RCT with statistically significant difference or narrow confidence intervals \\
\hline Level II & $\begin{array}{l}\text { - Low quality RCT (e.g., }<80 \% \text { follow-up) } \\
\text { - Systematic review of cohort studies } \\
\text { - Prospective comparative study }\end{array}$ \\
\hline Level III & $\begin{array}{l}\text { - Case-control study } \\
\text { - Systematic review of case-control study } \\
\text { Level IV }\end{array}$ \\
\hline Level V & $\begin{array}{l}\text { - Case series } \\
\text { - Poor quality case-control studies }\end{array}$ \\
\hline
\end{tabular}

Source: http://www.cebm.net/oxford-centre-evidence-based-medicine-levels-evidence-march-2009/

There are several studies examining the effectiveness of cochlear implants and other treatments in rehabilitating sound localization. Localization error is commonly used as an outcome measure to assess localization. Localization error is the mean difference (in degrees) between sound source localization and the source pointed out by the patient. Three studies reported sound localization, ${ }^{16-18}$ a sum of 26 patients.

Arndt et $\mathrm{al}^{16}$ compared sound localization using CROS, osseointegrated implant devices or cochlear implants six months after implantation in a cohort of 11 patients. Seven loudspeakers were placed in a semicircle in front of the patients, which were then asked to identify the speaker that was delivering the sound. Patients who received cochlear implants showed significantly less localization error compared to those in an unaided condition $(p=0.003)$, and patients with osseointegrated implant $(p=0.002)$, and patients with CROS hearing aid devices $(p=0.001)$.

Recently, Firszt et al ${ }^{17}$ reported that seven out of ten adults that had undergone cochlear implantation showed improvement in sound localization in the bimodal condition (CI plus hearing aid (HA) in better ear) compared with the HA-only condition ( $P \leq 0.05)$. Interestingly, these same seven had postlingual deafness in contrast to three who did not exhibit any improvement in sound localization and who presented with either prelingual or perilingual deafness.

Cardieux et $\mathrm{al}^{18}$ researched five patients with SSD submitted to $\mathrm{CI}$ and reported a significant enhancement in bimodal scores in three patients compared with those in the HA-only condition $(p<0.05)$. These results are in accordance with the outcomes in Firszt et al. ${ }^{17}$

\section{Effectiveness of Cochlear Implants in Improving Speech Perception}

Redundant information received by two independent acoustic sensors allows for summation and squelch. Binaural summation occurs when the same acoustic stimulus presents in both ears. The higher order auditory processing of redundant information provides $2-6 \mathrm{~dB}$ in signal threshold and is particularly beneficial in noisy environments. The squelch effect, on the other hand, represents a different form of higher order auditory processing, which helps to sort out meaningful sound from background noise, given that it is able to reduce noise ratio by $2-3 \mathrm{~dB} .{ }^{6,16,19}$ Cochlear implants allow for both an acoustic sensor and an electrical input to individuals' deaf side. Thus, if the auditory system can effectively combine this electrical signal with acoustic hearing in the opposite ear, patients that have undergone cochlear implantation will theoretically benefit from summation and squelch.

Seven studies reported on speech perception in patients with SSD and $\mathrm{CI}(\boldsymbol{n}=82){ }^{6,16-18,20-22}$ Different configurations have been used to assess overall speech understanding. In the following section, we decided to abbreviate sound (S) and noise $(\mathrm{N})$ followed by the direction: "HE" for sound or noise directed toward the hearing ear (better ear) and " $\mathrm{Cl}$ " for the cochlear implant side. The " 0 " is for 0 (zero) degrees azimuth.

Arndt et $\mathrm{al}^{16}$ evaluated speech perception using three conditions and compared CROS and bone-anchored hearing aid (BAHA) device recipients: first, sound and noise directed at the front of the patients head $\left(\mathrm{S}_{0} / \mathrm{N}_{0}\right)$, second, sound directed at normal hearing and noise directed at the deaf side $\left(\mathrm{S}_{\mathrm{HE}} / \mathrm{N}_{\mathrm{CI}}\right)$, and third, sound directed toward the deaf side and noise directed at the normal hearing ear $\left(\mathrm{S}_{\mathrm{Cl}} / \mathrm{N}_{\mathrm{HE}}\right)$. The results demonstrated that $\mathrm{CI}$ provided a statistically significant improvement in speech perception in the $\mathrm{S}_{\mathrm{HE}} / \mathrm{N}_{\mathrm{Cl}}$ and $\mathrm{S}_{\mathrm{CI}} /$ $\mathrm{N}_{\mathrm{HE}}$ configurations compared to those with CROS $(p=0.031$ and $p=0.03$ ) or osseointegrated implant devices $(p=0.023$ and $p=0.001$ ). The only configuration where $\mathrm{CI}$ was significantly superior to an unaided condition was $\mathrm{S}_{\mathrm{CI}} / \mathrm{N}_{\mathrm{HE}}$ ( $p=0.001)$. There was no significant difference in improvement between the groups when the noise was directed headon.

In agreement with Arndt et $\mathrm{al}^{16}$, Vermeire et $\mathrm{al}^{6}$ didńt find a significant outcome in the $\mathrm{S}_{0} / \mathrm{N}_{0}$ configuration. By contrast, they found a significant improvement in the binaural condition in the $\mathrm{S}_{\mathrm{Cl}} / \mathrm{N}_{0}$ configuration in both subgroups: the contralateral HA $(p=0.042)$ and normal-hearing ( $p=0.003)$ subgroups. Only the HA-subgroup experienced a significant benefit with the $\mathrm{Cl}$ activation $(p=0.031)$ in the $\mathrm{S}_{0} / \mathrm{N}_{\mathrm{CI}}$ configuration. 


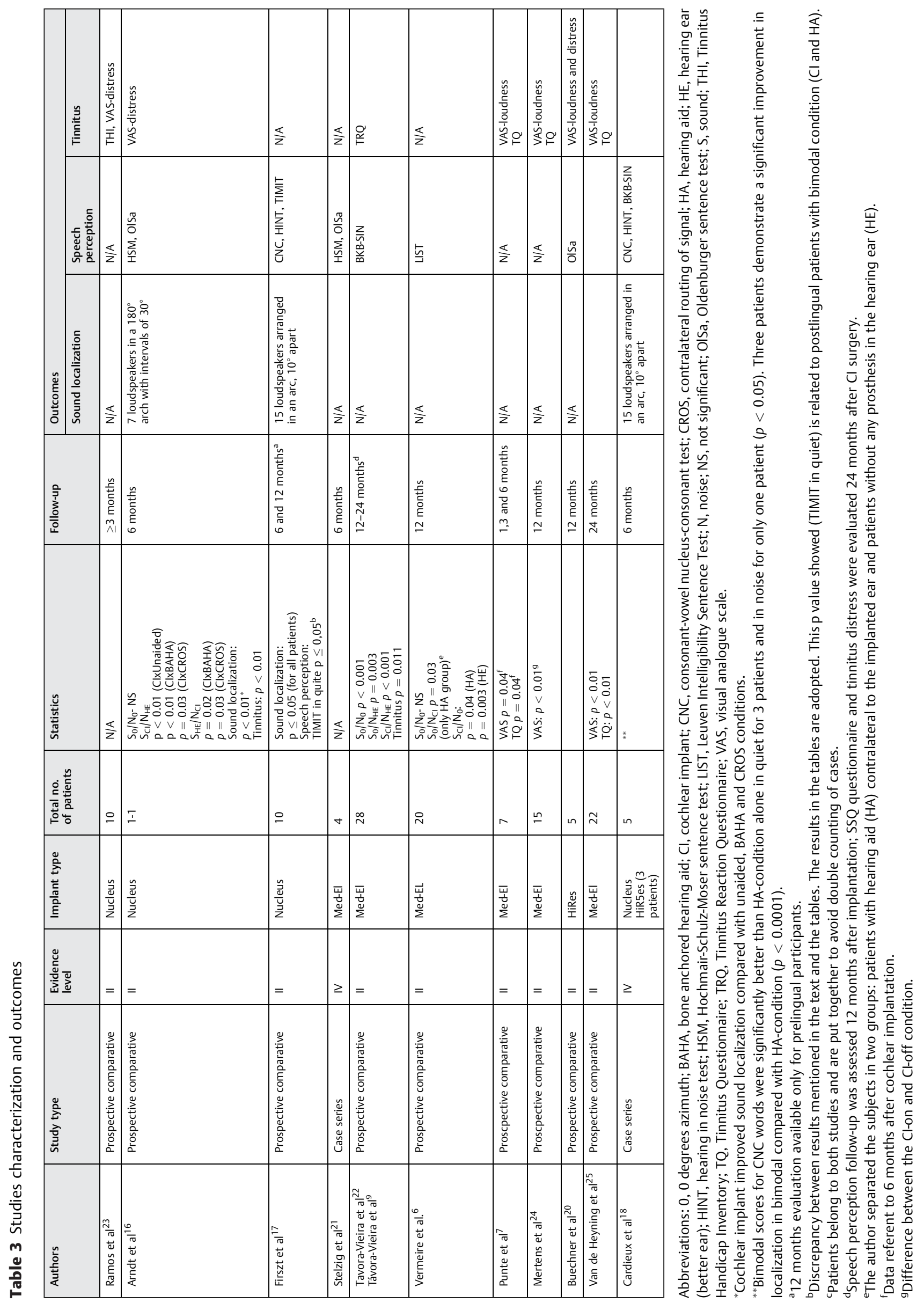


Firszt et al ${ }^{17}$ evaluated 10 patients with SSD or asymmetric hearing loss ( 7 postlingually and 3 prelingually) and demonstrated a significant improvement in speech perception in the $\mathrm{CI}$-only condition at the six-months interval for the following tests: Consonant-Vowel Nucleus-Consonant (CNC), $\mathrm{p} \leq 0.001$; Hearing in Noise Test (HINT), $\mathrm{p} \leq 0.001$; TIMIT in noise, $\mathrm{p} \leq$ 0.01 ; and TIMIT in quiet, $\mathrm{p} \leq 0.001$. The bimodal condition $(\mathrm{CI}+\mathrm{HA})$ showed a significantly better performance only in TIMIT sentences in a quiet environment $(p \leq 0.05)$. Only one of three pre-lingual subjects had open-set speech recognition with the $\mathrm{CI}$.

Another study designed by Buechner et $\mathrm{al}^{20}$ conducted with five patients showed that, for three patients $(p<0.01)$, $\mathrm{CI}$ led to a highly significantly improvement when noise was presented from the normal hearing side and speed from the front.

A case series conducted by Stelzig et $\mathrm{al}^{21}$ showed an improvement in speech perception measured by the Hochmair-Schulz-Moser sentence test (HSM) and the Oldenburger sentence test (OISa). All the patients' HSM scores were higher in the binaural condition. Their performance in the OlSa test was better when the noise signal was presented on the $\mathrm{CI}$ side and worse when the noise was presented on the normal hearing side. This study lacks statistic values.

Távora-Vieira et $\mathrm{al}^{22}$ showed a significant improvement in speech perception in noise scores when speech and noise were presented from the front and in the following arrangement: $\mathrm{S}_{0} / \mathrm{N}_{\mathrm{HE}}(p=0.003)$ and $\mathrm{S}_{\mathrm{CI}} / \mathrm{N}_{\mathrm{HE}}(p<0.001)$. The speech perception improved the most in the $\mathrm{S}_{\mathrm{CI}} / \mathrm{N}_{\mathrm{HE}}$, coinciding with the outcomes found by Arndt et $\mathrm{al}^{16}$ There was no significant interaction between age at implantation and duration of deafness. $^{22}$

Cardieux et $\mathrm{al}^{18}$ identified a significant improvement in performance in localization in the bimodal compared with HA-only condition for three of five patients $(p<0.001)$ studied.

\section{Tinnitus}

Some studies have evaluated the suppression or release of tinnitus after cochlear implantation. Seven studies analyzed herein report on tinnitus $(n=98)^{7,16,19,22-25}$ Six used a Visual Analog Scale (VAS) to assess tinnitus and two used questionnaires. Among the studies using VAS, four demonstrated a significant reduction of tinnitus loudness or distress after CI. ${ }^{7,16,24,25}$

Tavora-Vieira et al, ${ }^{22}$ in a very recent study from 2015, tracked twenty-eight patients for 24 months and demonstrated a significant decrease on tinnitus disturbance ( $p=0.011)$, which was measured using the Tinnitus Reaction Questionnaire (TRQ).

Ramos et $\mathrm{al}^{23}$ reported that, from a cohort of ten patients, two reported tinnitus suppression, seven experienced reduction in tinnitus intensity and one presented no change after cochlear implantation. The mean score for tinnitus retraining therapy (THI) fell from $72.1 \%$, preoperatively, to $14.3 \%$ at 3 months after cochlear implantation. The VAS showed a reduction from 7.9 points before surgery to 2.7 points at 3 months postoperatively.
Punte et $\mathrm{al}^{7}$ relayed tinnitus relief in patients with SSD after $\mathrm{CI}$. The authors observed a significant reduction of average VAS from 8.21 points $(S D=1.22)$ to $4.36(\mathrm{SD}=1.31)$ $(p=0.027)$ three months after activation of the CI. After six months, tinnitus loudness VAS decreases to 3.5 points $(p=0.042)$. Evaluation of tinnitus questionnaire (TQ) demonstrated a total score decrease (from 60.0 to 39.4) $(p=0.041)$ six months after implantation, denoting tinnitus improvement.

A significant reduction of tinnitus loudness was reported by Mertens et al, ${ }^{24}$ when they compared the CI-on and CI-off conditions. The mean VAS score was $7.2(\mathrm{SD}=2.6)$ in the Cl-off condition and declined to $3.4(\mathrm{SD}=2.5)$ in the $\mathrm{CI}$-on condition $(p<0.01)$. This study also reported an improvement in speech reception threshold (SRT) in the non-tinnitus ear when the cochlear implant was switched on $(p<0.01)$.

In a study by Van de Heyning et al, ${ }^{6,25}$ twenty-two patients in the cohort had tinnitus, of which three experienced suppression, 18 reported improvement and one reported no change.

Arndt et al $^{16}$ studied 11 patients with tinnitus in a cohort: six months after $\mathrm{CI}$ use, the median tinnitus intensity decreased significantly in the VAS score (from 5 to 0 ) when the CI was switched on ( $p=0.0078$ ). Five patients showed complete suppression of the tinnitus with the activated speech processor.

Three of five patients experienced suppression of the tinnitus in the study by Buechner et $\mathrm{al}^{20}$ However, no statistical data are shown for this study.

\section{Discussion}

Currently approved treatment solutions for unilateral hearing loss (contralateral routing of sound and osseointegrated implants) are effective in addressing the head shadow effect, but fail to provide psychoacoustic information to the deaf side (squelch and summation, which help to improve speech perception in noise).

A cochlear implant is the only option that provides earspecific information and, thus, potentially benefits SDD patients' bilateral listening. Furthermore, recognition in noise and sound localization are superior under binaural hearing. ${ }^{26}$ To date, overall selection criteria for cochlear implantation in SSD have not yet been established and the factors that may affect outcomes are unknown. Nevertheless, as long as familiarity with the cochlear implant device increases, there is a broadening of selection criteria for the surgery.

Based on that, we sought to review the literature regarding the effects of cochlear implantation on clinical outcomes, such as speech recognition, sound localization, and tinnitus, in patients with single-sided deafness. This systematic review is characterized by a critical appraisal and clear synthesis of the selected studies.

After rigorous evaluation, three studies could be analyzed in terms of sound localization. ${ }^{16-18}$ All of them presented statistical data, proving that sound localization is better in bimodal condition than in unaided or CROS/BAHA conditions. It is important to note that these outcomes refer to 
postlingual subjects. The only study that evaluated the outcomes of cochlear implantation in patients with prelingual onset of deafness was the one conducted by Firszt et al. ${ }^{17}$ They did not show any benefit from cochlear implantation in prelingual patients. This study suggests that, perhaps, there are some limitations in improving localization in patients with pre- or perilingual deafness. ${ }^{17}$ Our results on the outcomes described above are congruent with the results from Kamal et al ${ }^{27}$, Vlastarakos et $\mathrm{al}^{28}$, and Van Zon et al. ${ }^{29}$

Several studies describe improvement in speech perception; ${ }^{6,16-18,20-22}$ however, only four have shown consistent statistical data. ${ }^{6,16,17,22}$ All four used divergent parameters to measure outcome. Two of the studies ${ }^{16,22}$ found a significant improvement in speech understanding when sound is introduced at the cochlear implanted side and noise in ear with normal hearing, considered the most challenging situation in daily life. Only Távora-Vieira et $\mathrm{al}^{22}$ found statistically superior performance with a configuration with sound and noise introduced to the subjects front $\left(\mathrm{S}_{0} / \mathrm{N}_{0}\right)$. The abovementioned results are encouraging, in that they can be attributed to the squelch effect, meaning that the auditory system is able to process binaural signals after cochlear implantation. Recently, Vlastarakos et $\mathrm{al}^{28}$ and Van Zon et $\mathrm{al}^{29}$ reviewed speech perception in SSD patients, arriving at results consistent with our reports. ${ }^{28,29}$

Duration of deafness is a well-known factor affecting auditory performance in postlingual patients submitted to cochlear implantantion. ${ }^{30}$ Nonetheless, this study is the first review to analyze a study concerning this topic. Távora-Vieira et al., in 2015, were the first to investigate whether duration of deafness and age at implantation have an effect on the outcomes in postlingual patients. The study showed that these variables do not seem to affect the speech perception in noise or the improvement of tinnitus. ${ }^{22}$ Furthermore, their results, combined with $\mathrm{CI}$ acceptance, suggest that subjects with SSD are probably able to integrate the acoustic and electrical signals.

Cochlear implantation was used first in 2008 to treat tinnitus in patients with unilateral hearing loss. ${ }^{25}$ Reports of significant reduction of tinnitus after $\mathrm{CI}$ in patients with SSD confirm the effect of $\mathrm{CI}$ on treating unresponsive tinnitus (when tinnitus retraining therapy, sound therapy and drugs are not effective). ${ }^{7,16,22,24,25}$ The improvement of tinnitus can occur due several mechanisms: habituation, acoustic masking, direct stimulation of the cochlear nerve and organization of cortical pathways. ${ }^{21}$

Among seven studies that evaluated tinnitus relief or suppression, five presented statistically significant reductions of the symptom. ${ }^{7,16,22,24,25}$ The improvement of tinnitus distress or loudness after cochlear implantation supports the theory of auditory deafferentation, ${ }^{31,32}$ an outcome resulting from reafferentation by the restoring of auditory input. $^{33}$

In a recent meta-analysis analyzing only case series, Blasco and Redleaf ${ }^{34}$ found that cochlear implants had a statistically significant improvement in the severity of tinnitus. Van Zon et $\mathrm{al}^{29}$ analyzed six studies and reported a significant reduction of tinnitus distress in three of them.
Tokita et $\mathrm{al}^{3}$ and Vlastarakos et $\mathrm{al}^{28}$ found similar outcomes. These studies corroborate our view.

Finally, we would like to point the awkwardness in performing this systematic review, considering the large degree of heterogeneity in outcomes and subject groups among the studies. There is great variation concerning the duration and onset of deafness, and in some cases, no mention thereof. Moreover, studies diverge in their follow-up and, especially, the tests and parameters used to assess outcomes. These inconsistencies largely impede a straight-forward comparison between the studies.

\section{Final Comments}

We conclude that there is a large clinical heterogeneity among the studies that evaluated cochlear implantation in patients with unilateral hearing loss. Furthermore, there has yet to be a high level-of-evidence study performed concerning this question.

Outcomes regarding enhancement of sound localization, speech perception, and, mainly, improvement of tinnitus are promising indications as well; however, high quality studies are required before standardizing cochlear implantation as a treatment for single-sided deafness. Nonetheless, the results obtained up to this point from cochlear implantation in patients with single-sided deafness are encouraging in deeming this procedure a reasonable treatment. Given that the cochlear implant seems to bring greater benefits than contralateral routing of sound (CROS) and osseointegrated implants, it should be the first choice of treatment for patients with SSD in that which pertains to satisfactory selection criteria.

\section{References}

1 Baguley DM, Bird J, Humphriss RL, Prevost AT. The evidence base for the application of contralateral bone anchored hearing aids in acquired unilateral sensorineural hearing loss in adults. Clin Otolaryngol 2006;31(1):6-14

2 Kitterick PT, O’Donoghue GM, Edmondson-Jones M, et al. Comparison of the benefits of cochlear implantation versus contralateral routing of signal hearing aids in adult patients with singlesided deafness: study protocol for a prospective within-subject longitudinal trial. BMC Ear Nose Throat Disord 2014;14:7

3 Tokita J, Dunn C, Hansen MR. Cochlear implantation and singlesided deafness. Curr Opin Otolaryngol Head Neck Surg 2014; 22(5):353-358

4 Hol MK, Kunst SJ, Snik AF, Bosman AJ, Mylanus EA, Cremers CW. Bone-anchored hearing aids in patients with acquired and congenital unilateral inner ear deafness (Baha CROS): clinical evaluation of 56 cases. Ann Otol Rhinol Laryngol 2010;119(7):447-454

5 Wazen JJ, Van Ess MJ, Alameda J, Ortega C, Modisett M, Pinsky K. The Baha system in patients with single-sided deafness and contralateral hearing loss. Otolaryngol Head Neck Surg 2010; 142(4):554-559

6 Vermeire K, Van de Heyning P. Binaural hearing after cochlear implantation in subjects with unilateral sensorineural deafness and tinnitus. Audiol Neurootol 2009;14(3):163-171

7 Punte AK, De Ridder D, Van de Heyning P. On the necessity of full length electrical cochlear stimulation to suppress severe tinnitus in single-sided deafness. Hear Res 2013;295:24-29 
8 Firszt JB, Holden LK, Reeder RM, Waltzman SB, Arndt S. Auditory abilities after cochlear implantation in adults with unilateral deafness: a pilot study. Otol Neurotol 2012;33(8):1339-1346

9 Távora-Vieira D, Marino R, Krishnaswamy J, Kuthbutheen J, Rajan GP. Cochlear implantation for unilateral deafness with and without tinnitus: a case series. Laryngoscope 2013;123(5):1251-1255

10 Vermeire K, Nobbe A, Schleich P, Nopp P, Voormolen MH, Van de Heyning PH. Neural tonotopy in cochlear implants: an evaluation in unilateral cochlear implant patients with unilateral deafness and tinnitus. Hear Res 2008;245(1-2):98-106

11 Távora-Vieira D, Rajan GP. Cochlear implantation in children with congenital and noncongenital unilateral deafness: a case series. Otol Neurotol 2015;36(2):235-239

12 Hassepass F, Aschendorff A, Wesarg T, et al. Unilateral deafness in children: audiologic and subjective assessment of hearing ability after cochlear implantation. Otol Neurotol 2013;34(1):53-60

13 Hansen MR, Gantz BJ, Dunn C. Outcomes after cochlear implantation for patients with single-sided deafness, including those with recalcitrant Ménière's disease. Otol Neurotol 2013;34(9): 1681-1687

14 Perrott DR, Musicant AD. Dynamic minimum audible angle: binaural spatial acuity with moving sound sources. J Aud Res 1981;21(4):287-295

15 Soeta Y, Nakagawa S. Effects of the frequency of interaural time difference in the human brain. Neuroreport 2006;17(5):505-509

16 Arndt S, Aschendorff A, Laszig R, et al. Comparison of pseudobinaural hearing to real binaural hearing rehabilitation after cochlear implantation in patients with unilateral deafness and tinnitus. Otol Neurotol 2011;32(1):39-47

17 Firszt JB, Holden LK, Reeder RM, Cowdrey L, King S. Cochlear implantation in adults with asymmetric hearing loss. Ear Hear 2012;33(4):521-533

18 Cardieux JH, Firszt JB, Reeder RM. Cochlear implantation in nontraditional candidates: preliminary results in adolescents with asymmetric hearing loss. Otol Neurotol 2013;34(3):408-415

19 Schleich P, Nopp P, D'Haese P. Head shadow, squelch, and summation effects in bilateral users of the MED-EL COMBI 40/40+ cochlear implant. Ear Hear 2004;25(3):197-204

20 Buechner A, Brendel M, Lesinski-Schiedat A, et al. Cochlear implantation in unilateral deaf subjects associated with ipsilateral tinnitus. Otol Neurotol 2010;31(9):1381-1385

21 Stelzig Y, Jacob R, Mueller J. Preliminary speech recognition results after cochlear implantation in patients with unilateral hearing loss: a case series. J Med Case Reports 2011;5(5):343

22 Távora-Vieira D, Marino R, Acharya A, Rajan GP. The impact of cochlear implantation on speech understanding, subjective hear- ing performance, and tinnitus perception in patients with unilateral severe to profound hearing loss. Otol Neurotol 2015;36(3): 430-436

23 Ramos Á, Polo R, Masgoret E, et al. Cochlear implant in patients with sudden unilateral sensorineural hearing loss and associated tinnitus. Acta Otorrinolaringol Esp 2012;63(1):15-20

24 Mertens G, Kleine Punte A, De Ridder D, Van de Heyning P. Tinnitus in a single-sided deaf ear reduces speech reception in the nontinnitus ear. Otol Neurotol 2013;34(4):662-666

25 Van de Heyning P, Vermeire K, Diebl M, Nopp P, Anderson I, De Ridder D. Incapacitating unilateral tinnitus in single-sided deafness treated by cochlear implantation. Ann Otol Rhinol Laryngol 2008;117(9):645-652

26 Offeciers E, Morera C, Müller J, Huarte A, Shallop J, Cavallé L. International consensus on bilateral cochlear implants and bimodal stimulation. Acta Otolaryngol 2005;125(9): 918-919

27 Kamal SM, Robinson AD, Diaz RC. Cochlear implantation in singlesided deafness for enhancement of sound localization and speech perception. Curr Opin Otolaryngol Head Neck Surg 2012;20(5): 393-397

28 Vlastarakos PV, Nazos K, Tavoulari EF, Nikolopoulos TP. Cochlear implantation for single-sided deafness: the outcomes. An evidence-based approach. Eur Arch Otorhinolaryngol 2014;271(8): 2119-2126

29 van Zon A, Peters JPM, Stegeman I, Smit AL, Grolman W. Cochlear implantation for patients with single-sided deafness or asymmetrical hearing loss: a systematic review of the evidence. Otol Neurotol 2015;36(2):209-219

30 Blamey P, Artieres F, Başkent D, et al. Factors affecting auditory performance of postlinguistically deaf adults using cochlear implants: an update with 2251 patients. Audiol Neurootol 2013; 18(1):36-47

31 De Ridder D, Elgoyhen AB, Romo R, Langguth B. Phantom percepts: tinnitus and pain as persisting aversive memory networks. Proc Natl Acad Sci U S A 2011;108(20):8075-8080

32 Weisz N, Müller S, Schlee W, Dohrmann K, Hartmann T, Elbert T. The neural code of auditory phantom perception. J Neurosci 2007; 27(6):1479-1484

33 Sharma A, Martin K, Roland P, et al. P1 latency as a biomarker for central auditory development in children with hearing impairment. J Am Acad Audiol 2005;16(8):564-573

34 Blasco MA, Redleaf MI. Cochlear implantation in unilateral deafness improves tinnitus and speech comprehension: metaanalysis and systematic review. Otol Neurotol 2014;35(8): 1426-1432 